\title{
Two-component Analogue of Two-dimensional Long Wave-Short Wave Resonance Interaction Equations: A Derivation and Solutions
}

\author{
Yasuhiro Ohta $^{1}$, Ken-ichi Maruno ${ }^{2} \ddagger$ and Masayuki Oikawa ${ }^{3}$ \\ ${ }^{1}$ Department of Mathematics, Kobe University, Rokko, Kobe 657-8501, Japan \\ 2 Department of Mathematics, The University of Texas-Pan American, Edinburg, TX 78541 \\ 3 Research Institute for Applied Mechanics, Kyushu University, Kasuga, Fukuoka, \\ 816-8580, Japan
}

\begin{abstract}
The two-component analogue of two-dimensional long wave-short wave resonance interaction equations is derived in a physical setting. Wronskian solutions of the integrable two-component analogue of two-dimensional long wave-short wave resonance interaction equations are presented.
\end{abstract}

11 September 2018

PACS numbers: 02.30.Jr, 05.45.Yv

Keywords: Long wave-short wave resonance interaction equations, Vector solitons To be submitted to : J. Phys. A: Math. Gen.

\section{Introduction}

Recently, vector soliton equations (or coupled soliton equations) such as the vector Nonlinear Schrödinger (vNLS) equation have received so much attention in mathematical physics and nonlinear physics. Especially, the vNLS equation has been studied by several researchers from both mathematical and physical points of view [1-5]. It was pointed out that vector solitons can be used in the construction of logic gate [6-8]. It was also pointed out that the Yang-Baxter map is the key to understand the mathematical structure of logic gate based on vector solitons $[4,9,10]$.

Although there are many works about one-dimensional vector solitons, a mathematical work about two-dimensional vector solitons is still missing. For a more complete understanding mathematical structure of vector solitons, the study of two-dimensional vector solitons is very important.

In this paper, we derive a two-component analogue of two-dimensional long wave-short wave resonance interaction (2c-2d-LSRI) equations in a physical setting. We also present the Wronskian solution of the integrable 2c-2d-LSRI equations.

$\ddagger$ e-mail: kmaruno@utpa.edu 


\section{Derivation}

Consider the interaction of nonlinear dispersive waves on 3 channels, e.g. laser beams on some dispersive material. Suppose that the dispersion relations of these weakly nonlinear waves are

$$
\omega_{i}=\omega_{i}\left(k_{x, i}, k_{y, i}:\left|A_{1}\right|^{2},\left|A_{2}\right|^{2},\left|A_{3}\right|^{2}\right), \quad \text { for } \quad i=1,2,3
$$

where $\omega_{i}$ and $A_{i}$ are angular frequencies and amplitudes of each channel i, respectively Suppose that carrier wave is expressed by $\exp \left(i\left(k_{x, 0} x+k_{y, 0} y-\omega_{0} t\right)\right)$. Taylor expansion around $\mathbf{k}_{0}=\left(k_{x, 0}, k_{y, 0}\right), \omega_{0}$ and $\left|A_{i}\right|=0$ makes

$$
\begin{aligned}
& \omega_{i}-\omega_{0}=\left(\frac{\partial \omega_{i}}{\partial k_{x, i}}\right)_{0}\left(k_{x, i}-k_{x, 0}\right)+\left(\frac{\partial \omega_{i}}{\partial k_{y, i}}\right)_{0}\left(k_{y, i}-k_{y, 0}\right) \\
&+ \frac{1}{2}\left(\frac{\partial^{2} \omega_{i}}{\partial k_{x, i}^{2}}\right)_{0}\left(k_{x, i}-k_{x, 0}\right)^{2}+\frac{1}{2}\left(\frac{\partial^{2} \omega_{i}}{\partial k_{y, i}^{2}}\right)_{0}\left(k_{y, i}-k_{y, 0}\right)^{2} \\
&+\left(\frac{\partial^{2} \omega_{i}}{\partial k_{x, i} k_{y, i}}\right)_{0}\left(k_{x, i}-k_{x, 0}\right)\left(k_{y, i}-k_{y, 0}\right) \\
&+\left(\frac{\partial \omega_{i}}{\partial\left|A_{1}\right|^{2}}\right)_{0}\left|A_{1}\right|^{2}+\left(\frac{\partial \omega_{i}}{\partial\left|A_{2}\right|^{2}}\right)_{0}\left|A_{2}\right|^{2}+\left(\frac{\partial \omega_{i}}{\partial\left|A_{3}\right|^{2}}\right)_{0}\left|A_{3}\right|^{2}+\cdots, \\
& \text { for } i=1,2,3,
\end{aligned}
$$

where the subscript 0 of $(\quad)_{0}$ means setting $k_{x, i}=k_{x, 0}, k_{y, i}=k_{y, 0}, \omega_{i}=\omega_{0}$ and $\left|A_{i}\right|=0$. Replacing $\omega_{i}, k_{x, i}$ and $k_{y, i}$ to operators by the rules $\omega_{i}-\omega_{0} \sim \mathrm{i} \partial / \partial t, k_{x, i}-k_{x, 0} \sim-\mathrm{i} \partial / \partial x$, $k_{y, i}-k_{y, 0} \sim-\mathrm{i} \partial / \partial y$, and applying those equations to $A_{i}(x, y, t)$, we obtain

$$
\begin{aligned}
\mathrm{i} \frac{\partial A_{i}}{\partial t}+\mathrm{i}\left(\frac{\partial \omega_{i}}{\partial k_{x, i}}\right)_{0} \frac{\partial A_{i}}{\partial x}+\mathrm{i}\left(\frac{\partial \omega_{i}}{\partial k_{y, i}}\right)_{0} \frac{\partial A_{i}}{\partial y} \\
+\frac{1}{2}\left(\frac{\partial^{2} \omega_{i}}{\partial k_{x, i}^{2}}\right)_{0} \frac{\partial^{2} A_{i}}{\partial x^{2}}+\frac{1}{2}\left(\frac{\partial^{2} \omega_{i}}{\partial k_{y, i}^{2}}\right)_{0} \frac{\partial^{2} A_{i}}{\partial y^{2}}+\left(\frac{\partial^{2} \omega_{i}}{\partial k_{x, i} \partial k_{y, i}}\right)_{0} \frac{\partial^{2} A_{i}}{\partial x \partial y} \\
-\left(\frac{\partial \omega_{i}}{\partial\left|A_{1}\right|^{2}}\right)_{0}\left|A_{1}\right|^{2} A_{i}-\left(\frac{\partial \omega_{i}}{\partial\left|A_{2}\right|^{2}}\right)_{0}\left|A_{2}\right|^{2} A_{i}-\left(\frac{\partial \omega_{i}}{\partial\left|A_{3}\right|^{2}}\right)_{0}\left|A_{3}\right|^{2} A_{i}=0, \\
\text { for } i=1,2,3 .
\end{aligned}
$$

By the transformation of coordinate

$$
x^{\prime}=x-\left(\frac{\partial \omega_{3}}{\partial k_{x, 3}}\right)_{0} t, \quad y^{\prime}=y-\left(\frac{\partial \omega_{3}}{\partial k_{y, 3}}\right)_{0} t, \quad t^{\prime}=t
$$

we obtain

$$
\begin{aligned}
& i \frac{\partial A_{1}}{\partial t}+i v_{x, 1} \frac{\partial A_{1}}{\partial x}+i v_{y, 1} \frac{\partial A_{1}}{\partial y} \\
& \quad+\frac{1}{2}\left(\frac{\partial^{2} \omega_{1}}{\partial k_{x, 1}^{2}}\right)_{0} \frac{\partial^{2} A_{1}}{\partial x^{2}}+\frac{1}{2}\left(\frac{\partial^{2} \omega_{1}}{\partial k_{y, 1}^{2}}\right)_{0} \frac{\partial^{2} A_{1}}{\partial y^{2}}+\left(\frac{\partial^{2} \omega_{1}}{\partial k_{x, 1} \partial k_{y, 1}}\right)_{0} \frac{\partial^{2} A_{1}}{\partial x \partial y} \\
& \quad-\left(\frac{\partial \omega_{1}}{\partial\left|A_{1}\right|^{2}}\right)_{0}\left|A_{1}\right|^{2} A_{1}-\left(\frac{\partial \omega_{1}}{\partial\left|A_{2}\right|^{2}}\right)_{0}\left|A_{2}\right|^{2} A_{1}-\left(\frac{\partial \omega_{1}}{\partial\left|A_{3}\right|^{2}}\right)_{0}\left|A_{3}\right|^{2} A_{1}=0, \\
& i \frac{\partial A_{2}}{\partial t}+i v_{x, 2} \frac{\partial A_{2}}{\partial x}+i v_{y, 2} \frac{\partial A_{2}}{\partial y}
\end{aligned}
$$




$$
\begin{gathered}
+\frac{1}{2}\left(\frac{\partial^{2} \omega_{2}}{\partial k_{x, 2}^{2}}\right)_{0} \frac{\partial^{2} A_{2}}{\partial x^{2}}+\frac{1}{2}\left(\frac{\partial^{2} \omega_{2}}{\partial k_{y, 2}^{2}}\right)_{0} \frac{\partial^{2} A_{2}}{\partial y^{2}}+\left(\frac{\partial^{2} \omega_{2}}{\partial k_{x, 2} \partial k_{y, 2}}\right)_{0} \frac{\partial^{2} A_{2}}{\partial x \partial y} \\
-\left(\frac{\partial \omega_{2}}{\partial\left|A_{1}\right|^{2}}\right)_{0}\left|A_{1}\right|^{2} A_{2}-\left(\frac{\partial \omega_{2}}{\partial\left|A_{2}\right|^{2}}\right)_{0}\left|A_{2}\right|^{2} A_{2}-\left(\frac{\partial \omega_{2}}{\partial\left|A_{3}\right|^{2}}\right)_{0}\left|A_{3}\right|^{2} A_{2}=0, \\
i \frac{\partial A_{3}}{\partial t}+\frac{1}{2}\left(\frac{\partial^{2} \omega_{3}}{\partial k_{x, 3}^{2}}\right)_{0} \frac{\partial^{2} A_{3}}{\partial x^{2}}+\frac{1}{2}\left(\frac{\partial^{2} \omega_{3}}{\partial k_{y, 3}^{2}}\right)_{0} \frac{\partial^{2} A_{3}}{\partial y^{2}}+\left(\frac{\partial^{2} \omega_{3}}{\partial k_{x, 3} \partial k_{y, 3}}\right)_{0} \frac{\partial^{2} A_{3}}{\partial x \partial y} \\
-\left(\frac{\partial \omega_{3}}{\partial\left|A_{1}\right|^{2}}\right)_{0}\left|A_{1}\right|^{2} A_{3}-\left(\frac{\partial \omega_{3}}{\partial\left|A_{2}\right|^{2}}\right)_{0}\left|A_{2}\right|^{2} A_{3}-\left(\frac{\partial \omega_{3}}{\partial\left|A_{3}\right|^{2}}\right)_{0}\left|A_{3}\right|^{2} A_{3}=0,
\end{gathered}
$$

where

$$
\begin{aligned}
& v_{x, 1}=\left(\frac{\partial \omega_{1}}{\partial k_{x, 1}}\right)_{0}-\left(\frac{\partial \omega_{3}}{\partial k_{x, 3}}\right)_{0}, \quad v_{y, 1}=\left(\frac{\partial \omega_{1}}{\partial k_{y, 1}}\right)_{0}-\left(\frac{\partial \omega_{3}}{\partial k_{y, 3}}\right)_{0}, \\
& v_{x, 2}=\left(\frac{\partial \omega_{2}}{\partial k_{x, 2}}\right)_{0}-\left(\frac{\partial \omega_{3}}{\partial k_{x, 3}}\right)_{0}, \quad v_{y, 2}=\left(\frac{\partial \omega_{2}}{\partial k_{y, 2}}\right)_{0}-\left(\frac{\partial \omega_{3}}{\partial k_{y, 3}}\right)_{0},
\end{aligned}
$$

and $x^{\prime}, y^{\prime}, t^{\prime}$ are replaced by $x, y, t$. This is a generalization of the vector Nonlinear Schrödinger equation (Manakov system) [2, 11].

Now, consider the situation in which $v_{x, 1}$ and $v_{x, 2}$ are neglected. For notational convenience, we rewrite the above equations as

$$
\begin{aligned}
& i \frac{\partial A_{1}}{\partial t}+i v_{y, 1} \frac{\partial A_{1}}{\partial y}+\alpha_{1} \frac{\partial^{2} A_{1}}{\partial x^{2}}+\alpha_{2} \frac{\partial^{2} A_{1}}{\partial y^{2}}+\alpha_{3} \frac{\partial^{2} A_{1}}{\partial x \partial y} \\
& +\alpha_{4}\left|A_{1}\right|^{2} A_{1}+\alpha_{5}\left|A_{2}\right|^{2} A_{1}+\alpha_{6}\left|A_{3}\right|^{2} A_{1}=0, \\
& i \frac{\partial A_{2}}{\partial t}+i v_{y, 2} \frac{\partial A_{2}}{\partial y}+\beta_{1} \frac{\partial^{2} A_{2}}{\partial x^{2}}+\beta_{2} \frac{\partial^{2} A_{2}}{\partial y^{2}}+\beta_{3} \frac{\partial^{2} A_{2}}{\partial x \partial y} \\
& \quad+\beta_{4}\left|A_{1}\right|^{2} A_{2}+\beta_{5}\left|A_{2}\right|^{2} A_{2}+\beta_{6}\left|A_{3}\right|^{2} A_{2}=0, \\
& i \frac{\partial A_{3}}{\partial t}+\gamma_{1} \frac{\partial^{2} A_{3}}{\partial x^{2}}+\gamma_{2} \frac{\partial^{2} A_{3}}{\partial y^{2}}+\gamma_{3} \frac{\partial^{2} A_{3}}{\partial x \partial y} \\
& \quad+\gamma_{4}\left|A_{1}\right|^{2} A_{3}+\gamma_{5}\left|A_{2}\right|^{2} A_{3}+\gamma_{6}\left|A_{3}\right|^{2} A_{3}=0 .
\end{aligned}
$$

Assume that the channel 3 is normal dispersion and the channels 1 and 2 are anomalous dispersion. We study the dark pulses generated in the channel $3:[12]$

$\left.A_{1}=\psi_{1} \exp \left(\mathrm{i} \delta_{1} t\right), \quad A_{2}=\psi_{2} \exp \left(\mathrm{i} \delta_{2} t\right), \quad A_{3}=\left(u_{0}+a(x, y, t)\right) \exp (\mathrm{i} \Gamma t+\mathrm{i} \phi(x, y, t))\right)$, $\delta_{1}=-\left(\frac{\partial \omega_{1}}{\partial\left|A_{3}\right|^{2}}\right)_{0} u_{0}^{2}, \quad \delta_{2}=-\left(\frac{\partial \omega_{2}}{\partial\left|A_{3}\right|^{2}}\right)_{0} u_{0}^{2}, \quad \Gamma=-\left(\frac{\partial \omega_{3}}{\partial\left|A_{3}\right|^{2}}\right)_{0} u_{0}^{2}$,

where $a$ and $\psi_{i}(i=1,2)$ are small. Substituting these into equations (2.6)-(2.8), we obtain

$$
\begin{aligned}
& \frac{\partial a}{\partial t}+\gamma_{1} u_{0} \frac{\partial^{2} \phi}{\partial x^{2}}+\gamma_{2} u_{0} \frac{\partial^{2} \phi}{\partial y^{2}}+\gamma_{3} u_{0} \frac{\partial^{2} \phi}{\partial x \partial y}=0, \\
& -u_{0} \frac{\partial \phi}{\partial t}+\gamma_{1} \frac{\partial^{2} a}{\partial x^{2}}+\gamma_{2} \frac{\partial^{2} a}{\partial y^{2}}+\gamma_{3} \frac{\partial^{2} a}{\partial x \partial y} \\
& \quad+\gamma_{4} u_{0}\left|\psi_{1}\right|^{2}+\gamma_{5} u_{0}\left|\psi_{2}\right|^{2}+3 \gamma_{6} u_{0}^{2} a=0, \\
& i \frac{\partial \psi_{1}}{\partial t}+i v_{y, 1} \frac{\partial \psi_{1}}{\partial y}+\alpha_{1} \frac{\partial^{2} \psi_{1}}{\partial x^{2}}+\alpha_{2} \frac{\partial^{2} \psi_{1}}{\partial y^{2}}+\alpha_{3} \frac{\partial^{2} \psi_{1}}{\partial x \partial y}
\end{aligned}
$$




$$
\begin{gathered}
+\alpha_{4}\left|\psi_{1}\right|^{2} \psi_{1}+\alpha_{5}\left|\psi_{2}\right|^{2} \psi_{1}+2 \alpha_{6} u_{0} a \psi_{1}=0 \\
i \frac{\partial \psi_{2}}{\partial t}+i v_{y, 2} \frac{\partial \psi_{2}}{\partial y}+\beta_{1} \frac{\partial^{2} \psi_{2}}{\partial x^{2}}+\beta_{2} \frac{\partial^{2} \psi_{2}}{\partial y^{2}}+\beta_{3} \frac{\partial^{2} \psi_{2}}{\partial x \partial y} \\
\quad+\beta_{4}\left|\psi_{1}\right|^{2} \psi_{2}+\beta_{5}\left|\psi_{2}\right|^{2} \psi_{2}+2 \beta_{6} u_{0} a \psi_{2}=0 .
\end{gathered}
$$

Assume that the $y$-dependency of $\phi$ can be neglected, i.e. we can neglect $\phi_{y}$ and $\phi_{y y}$. Then eq.(2.9) reduces to

$$
\frac{\partial a}{\partial t}+\gamma_{1} u_{0} \frac{\partial^{2} \phi}{\partial x^{2}}=0
$$

i.e.,

$$
\frac{\partial^{2} \phi}{\partial x^{2}}=-\frac{1}{\gamma_{1} u_{0}} \frac{\partial a}{\partial t} .
$$

Substitute this into eq.(2.10), we have

$$
\begin{aligned}
\frac{\partial^{2} a}{\partial t^{2}}+ & 3 \gamma_{1} \gamma_{6} u_{0}^{2} \frac{\partial^{2} a}{\partial x^{2}}+\gamma_{1}^{2} \frac{\partial^{4} a}{\partial x^{4}}+\gamma_{1} \gamma_{2} \frac{\partial^{4} a}{\partial x^{2} \partial y^{2}}+\gamma_{1} \gamma_{3} \frac{\partial^{4} a}{\partial x^{3} \partial y} \\
& +\gamma_{1} \frac{\partial^{2}}{\partial x^{2}}\left(\gamma_{4} u_{0}\left|\psi_{1}\right|^{2}+\gamma_{5} u_{0}\left|\psi_{2}\right|^{2}\right)=0 .
\end{aligned}
$$

By

$$
t^{\prime}=\varepsilon t, \quad x^{\prime}=\varepsilon^{1 / 2}(x+c t), \quad y^{\prime}=\varepsilon y,
$$

( $c=3 \gamma_{1} \gamma_{6} u_{0}^{2}$ ) with $a=\varepsilon a_{0}, \psi_{1}=\varepsilon^{3 / 4} \Phi_{1}, \psi_{2}=\varepsilon^{3 / 4} \Phi_{2}$ ( $\varepsilon$ is small), we obtain equations of lowest order of $\varepsilon$

$$
\begin{aligned}
& 2 c \frac{\partial^{2} a}{\partial x \partial t}+\gamma_{1} \frac{\partial^{2}}{\partial x^{2}}\left(\gamma_{4} u_{0}\left|\psi_{1}\right|^{2}+\gamma_{5} u_{0}\left|\psi_{2}\right|^{2}\right)=0 \\
& i \frac{\partial \psi_{1}}{\partial t}+i v_{y, 1} \frac{\partial \psi_{1}}{\partial y}+\alpha_{1} \frac{\partial^{2} \psi_{1}}{\partial x^{2}}+2 \alpha_{6} u_{0} a \psi_{1}=0 \\
& i \frac{\partial \psi_{2}}{\partial t}+i v_{y, 2} \frac{\partial \psi_{2}}{\partial y}+\beta_{1} \frac{\partial^{2} \psi_{2}}{\partial x^{2}}+2 \beta_{6} u_{0} a \psi_{2}=0
\end{aligned}
$$

Here we again have disregarded the primes and have replaced $a_{0}, \Phi_{1}$ and $\Phi_{2}$ with $a, \psi_{1}$ and $\psi_{2}$. The first equation leads to

$$
2 c \frac{\partial a}{\partial t}+\gamma_{1} \frac{\partial}{\partial x}\left(\gamma_{4} u_{0}\left|\psi_{1}\right|^{2}+\gamma_{5} u_{0}\left|\psi_{2}\right|^{2}\right)=0 .
$$

This system is nothing but the 2-component analogue of 2-dimensional analogue of the long wave-short wave resonance interaction (2c-2d-LSRI) equations[13-16]. Note that the special case of coefficients $\left(v_{y, 1}=v_{y, 2}, \alpha_{1}=\beta_{1}, \alpha_{6}=\beta_{6}, \gamma_{4}=\gamma_{5}\right)$ is integrable. In the onecomponent integrable case, several solutions have been presented [15, 17-19]. In Ref.[20], some solutions of matrix generalization were discussed. In the next section, we will present the determinant formula of $N$-soliton solution for the integrable 2c-2d-LSRI equations.

\section{Soliton Solutions}

We study the soliton solutions of an integrable case of the 2c-2d-LSRI equations

$$
\begin{aligned}
& i\left(S_{t}^{(1)}+S_{y}^{(1)}\right)-S_{x x}^{(1)}+L S^{(1)}=0, \\
& i\left(S_{t}^{(2)}+S_{y}^{(2)}\right)-S_{x x}^{(2)}+L S^{(2)}=0, \\
& L_{t}=2\left(\left|S^{(1)}\right|^{2}\right)_{x}+2\left(\left|S^{(2)}\right|^{2}\right)_{x} .
\end{aligned}
$$


By the dependent variable transformation

$$
S^{(1)}=\frac{G}{F}, \quad S^{(2)}=\frac{H}{F}, \quad L=-2 \frac{\partial^{2}}{\partial x^{2}} \log F,
$$

we have three bilinear equations

$$
\begin{aligned}
& {\left[i\left(D_{t}+D_{y}\right)-D_{x}^{2}\right] G \cdot F=0,} \\
& {\left[i\left(D_{t}+D_{y}\right)-D_{x}^{2}\right] H \cdot F=0,} \\
& \left(D_{t} D_{x}-2 c\right) F \cdot F+2 G G^{*}+2 H H^{*}=0 .
\end{aligned}
$$

Here we set $c=0$ which means we consider the bright-type soliton solutions. These bilinear equations belong to the three-component KP hierarchy [21-24]. We can construct soliton solutions in which the number of solitons in channel $i$ is $N_{i}(i=1,2,3)$. We call this solution $\left(N_{1}, N_{2}, N_{3}\right)$-soliton solution.

\section{Wronskian form of the bright type $(N, M, N+M)$-soliton solutions}

\section{Let}

$$
\begin{aligned}
& \tau_{n m}^{N M}= \\
& \left|\begin{array}{cccccccccccc}
\varphi_{1} & \varphi_{1}^{(1)} & \cdots & \varphi_{1}^{(N+M-1+n+m)} & \psi_{1} & \psi_{1}^{(1)} & \cdots & \psi_{1}^{(N-1-n)} & 0 & 0 & \cdots & 0 \\
\varphi_{2} & \varphi_{2}^{(1)} & \cdots & \varphi_{2}^{(N+M-1+n+m)} & \psi_{2} & \psi_{2}^{(1)} & \cdots & \psi_{2}^{(N-1-n)} & 0 & 0 & \cdots & 0 \\
\vdots & \vdots & & \vdots & \vdots & \vdots & & \vdots & \vdots & \vdots & & \vdots \\
\varphi_{2 N} & \varphi_{2 N}^{(1)} & \cdots & \varphi_{2 N}^{(N+M-1+n+m)} & \psi_{2 N} & \psi_{2 N}^{(1)} & \cdots & \psi_{2 N}^{(N-1-n)} & 0 & 0 & \cdots & 0 \\
\phi_{1} & \phi_{1}^{(1)} & \cdots & \phi_{1}^{(N+M-1+n+m)} & 0 & 0 & \cdots & 0 & \chi_{1} & \chi_{1}^{(1)} & \cdots & \chi_{1}^{(M-1-m)} \\
\phi_{2} & \phi_{2}^{(1)} & \cdots & \phi_{2}^{(N+M-1+n+m)} & 0 & 0 & \cdots & 0 & \chi_{2} & \chi_{2}^{(1)} & \cdots & \chi_{2}^{(M-1-m)} \\
\vdots & \vdots & & \vdots & \vdots & \vdots & & \vdots & \vdots & \vdots & & \vdots \\
\phi_{2 M} & \phi_{2 M}^{(1)} & \cdots & \phi_{2 M}^{(N+M-1+n+m)} & 0 & 0 & \cdots & 0 & \chi_{2 M} & \chi_{2 M}^{(1)} & \cdots & \chi_{2 M}^{(M-1-m)}
\end{array}\right| \\
& \varphi_{i}=e^{\xi_{i}}, \quad \xi_{i}=p_{i} x_{1}+p_{i}^{2} x_{2}, \quad \text { for } i=1,2, \cdots, N \\
& \varphi_{N+i}=e^{-\xi_{i}^{*}}, \quad-\xi_{i}^{*}=-p_{i}^{*} x_{1}+\left(-p_{i}^{*}\right)^{2} x_{2}, \quad \text { for } i=1,2, \cdots, N \\
& \phi_{i}=e^{\theta_{i}}, \quad \theta_{i}=s_{i} x_{1}+s_{i}^{2} x_{2}, \quad \text { for } i=1,2, \cdots, M \\
& \phi_{M+i}=e^{-\theta_{i}^{*}}, \quad-\theta_{i}^{*}=-s_{i}^{*} x_{1}+\left(-s_{i}^{*}\right)^{2} x_{2}, \quad \text { for } i=1,2, \cdots, M \\
& \psi_{i}=a_{i} e^{\eta_{i}}, \quad \eta_{i}=q_{i} y_{1}+\eta_{i 0}, \quad \text { for } i=1,2, \cdots, N \\
& \psi_{N+i}=a_{N+i} e^{-\eta_{i}^{*}}, \quad-\eta_{i}^{*}=-q_{i}^{*} y_{1}-\eta_{i 0}^{*}, \quad \text { for } i=1,2, \cdots, N \\
& \chi_{i}=b_{i} e^{\zeta_{i}}, \quad \zeta_{i}=r_{i} z_{1}+\zeta_{i 0}, \quad \text { for } i=1,2, \cdots, M \\
& \chi_{M+i}=b_{M+i} e^{-\zeta_{i}^{*}}, \quad-\zeta_{i}^{*}=-r_{i}^{*} z_{1}-\zeta_{i 0}^{*}, \quad \text { for } i=1,2, \cdots, M \\
& a_{i}=\left(\prod_{\substack{k=1 \\
k \neq i}}^{N} \frac{p_{k}-p_{i}}{q_{k}-q_{i}}\right)\left(\prod_{l=1}^{M}\left(s_{l}-p_{i}\right)\right), \quad \text { for } i=1,2, \cdots, N \\
& a_{N+i}=\varepsilon_{i}\left(\prod_{k=1}^{N} \frac{p_{k}+p_{i}^{*}}{q_{k}+q_{i}^{*}}\right)\left(\prod_{l=1}^{M}\left(s_{l}+p_{i}^{*}\right)\right), \quad \text { for } i=1,2, \cdots, N \\
& b_{i}=\left(\prod_{k=1}^{N}\left(p_{k}-s_{i}\right)\right)\left(\prod_{\substack{l=1 \\
l \neq i}}^{M} \frac{s_{l}-s_{i}}{r_{l}-r_{i}}\right), \quad \text { for } i=1,2, \cdots, M \\
& b_{M+i}=\delta_{i}\left(\prod_{k=1}^{N}\left(p_{k}+s_{i}^{*}\right)\right)\left(\prod_{l=1}^{M} \frac{s_{l}+s_{i}^{*}}{r_{l}+r_{i}^{*}}\right), \quad \text { for } i=1,2, \cdots, M
\end{aligned}
$$




$$
\varepsilon_{i}= \pm 1, \quad \delta_{i}= \pm 1
$$

where ${ }^{*}$ means complex conjugate and $p_{i}, q_{i}(1 \leq i \leq N)$ and $s_{i}, r_{i}(1 \leq i \leq M)$ are complex wave numbers, and $\eta_{i 0}(1 \leq i \leq N)$ and $\zeta_{i 0}(1 \leq i \leq M)$ are complex phase parameters. In order to obtain regular solutions, we have to choose appropriate sign for $\varepsilon_{i}$ and $\delta_{i}$, which depend on parameters $p_{i}, q_{i}, r_{i}, s_{i}$. We take

$$
x_{1}=x, \quad x_{2}=-i y, \quad y_{1}=y-t, \quad z_{1}=y-t,
$$

where $x, y$ and $t$ are real, (i.e. $x_{1}, y_{1}$ and $z_{1}$ are real and $x_{2}$ is pure imaginary).

Let

$$
f=\tau_{00}, \quad g=\tau_{10}, \quad \bar{g}=\tau_{-1,0}, \quad h=\tau_{01}, \quad \bar{h}=\tau_{0,-1} .
$$

These tau-functions satisfy the condition

$$
\left(\frac{g}{f}\right)^{*}=\frac{\bar{g}}{f}, \quad\left(\frac{h}{f}\right)^{*}=\frac{\bar{h}}{f},
$$

$f \mathcal{G}:$ real .

where $\mathcal{G}$ is an exponential factor which is a gauge function (see Appendix). Let $F=f \mathcal{G}$, $G=g \mathcal{G}, G^{*}=\bar{g} \mathcal{G}, H=h \mathcal{G}, H^{*}=\bar{h} \mathcal{G}$. The functions $F, G$ and $H$ satisfy the bilinear equations (3.5)-(3.7) and reality of $F$ and complex conjugacy of $G$ and $H$. The function $L=-2 \frac{\partial^{2}}{\partial x^{2}} \log F$ represents $N+M$-soliton solution, $S_{1}=G / F$ represents $N$-soliton solution, and $S_{2}=H / F$ represents $M$-soliton solution.

\section{$(1,1,2)$-soliton solution}

The $\tau$-functions of $(1,1,2)$-soliton solution are the following:

$$
\begin{aligned}
& f=\left|\begin{array}{cccc}
\varphi_{1} & \varphi_{1}^{(1)} & \psi_{1} & 0 \\
\varphi_{2} & \varphi_{2}^{(1)} & \psi_{2} & 0 \\
\phi_{1} & \phi_{1}^{(1)} & 0 & \chi_{1} \\
\phi_{2} & \phi_{2}^{(1)} & 0 & \chi_{2}
\end{array}\right|, \\
& g=\left|\begin{array}{llll}
\varphi_{1} & \varphi_{1}^{(1)} & \varphi_{1}^{(2)} & 0 \\
\varphi_{2} & \varphi_{2}^{(1)} & \varphi_{2}^{(2)} & 0 \\
\phi_{1} & \phi_{1}^{(1)} & \phi_{1}^{(2)} & \chi_{1} \\
\phi_{2} & \phi_{2}^{(1)} & \phi_{2}^{(2)} & \chi_{2}
\end{array}\right|, \quad \bar{g}=\left|\begin{array}{cccc}
\varphi_{1} & \psi_{1} & \psi_{1}^{(1)} & 0 \\
\varphi_{2} & \psi_{2} & \psi_{2}^{(1)} & 0 \\
\phi_{1} & 0 & 0 & \chi_{1} \\
\phi_{2} & 0 & 0 & \chi_{2}
\end{array}\right| \\
& h=\left|\begin{array}{llll}
\varphi_{1} & \varphi_{1}^{(1)} & \varphi_{1}^{(2)} & \psi_{1} \\
\varphi_{2} & \varphi_{2}^{(1)} & \varphi_{2}^{(2)} & \psi_{2} \\
\phi_{1} & \phi_{1}^{(1)} & \phi_{1}^{(2)} & 0 \\
\phi_{2} & \phi_{2}^{(1)} & \phi_{2}^{(2)} & 0
\end{array}\right|, \quad \bar{h}=\left|\begin{array}{cccc}
\varphi_{1} & \psi_{1} & 0 & 0 \\
\varphi_{2} & \psi_{2} & 0 & 0 \\
\phi_{1} & 0 & \chi_{1} & \chi_{1}^{(1)} \\
\phi_{2} & 0 & \chi_{2} & \chi_{2}^{(1)}
\end{array}\right| \\
& \varphi_{1}=e^{\xi_{1}}, \quad \xi_{1}=p_{1} x_{1}+p_{1}^{2} x_{2}, \quad \varphi_{2}=e^{-\xi_{1}^{*}}, \quad-\xi_{1}^{*}=-p_{1}^{*} x_{1}+\left(-p_{1}^{*}\right)^{2} x_{2}, \\
& \phi_{1}=e^{\theta_{1}}, \quad \theta_{1}=s_{1} x_{1}+s_{1}^{2} x_{2}, \quad \phi_{2}=e^{-\theta_{1}^{*}}, \quad-\theta_{1}^{*}=-s_{1}^{*} x_{1}+\left(-s_{1}^{*}\right)^{2} x_{2}, \\
& \psi_{1}=a_{1} e^{\eta_{1}}, \quad \eta_{1}=q_{1} y_{1}+\eta_{10}, \quad \psi_{2}=a_{2} e^{-\eta_{1}^{*}}, \quad-\eta_{1}^{*}=-q_{1}^{*} y_{1}-\eta_{10}^{*}, \\
& \chi_{1}=b_{1} e^{\zeta_{1}}, \quad \zeta_{1}=r_{1} z_{1}+\zeta_{10}, \quad \chi_{2}=b_{2} e^{-\zeta_{1}^{*}}, \quad-\zeta_{1}^{*}=-r_{1}^{*} z_{1}-\zeta_{10}^{*}, \\
& a_{1}=s_{1}-p_{1}, \quad b_{1}=p_{1}-s_{1}, \quad a_{2}=\varepsilon\left(s_{1}+p_{1}^{*}\right) \frac{p_{1}+p_{1}^{*}}{q_{1}+q_{1}^{*}}, \quad b_{2}=\delta\left(s_{1}^{*}+p_{1}\right) \frac{s_{1}+s_{1}^{*}}{r_{1}+r_{1}^{*}}, \\
& \varepsilon= \pm 1, \quad \delta= \pm 1
\end{aligned}
$$



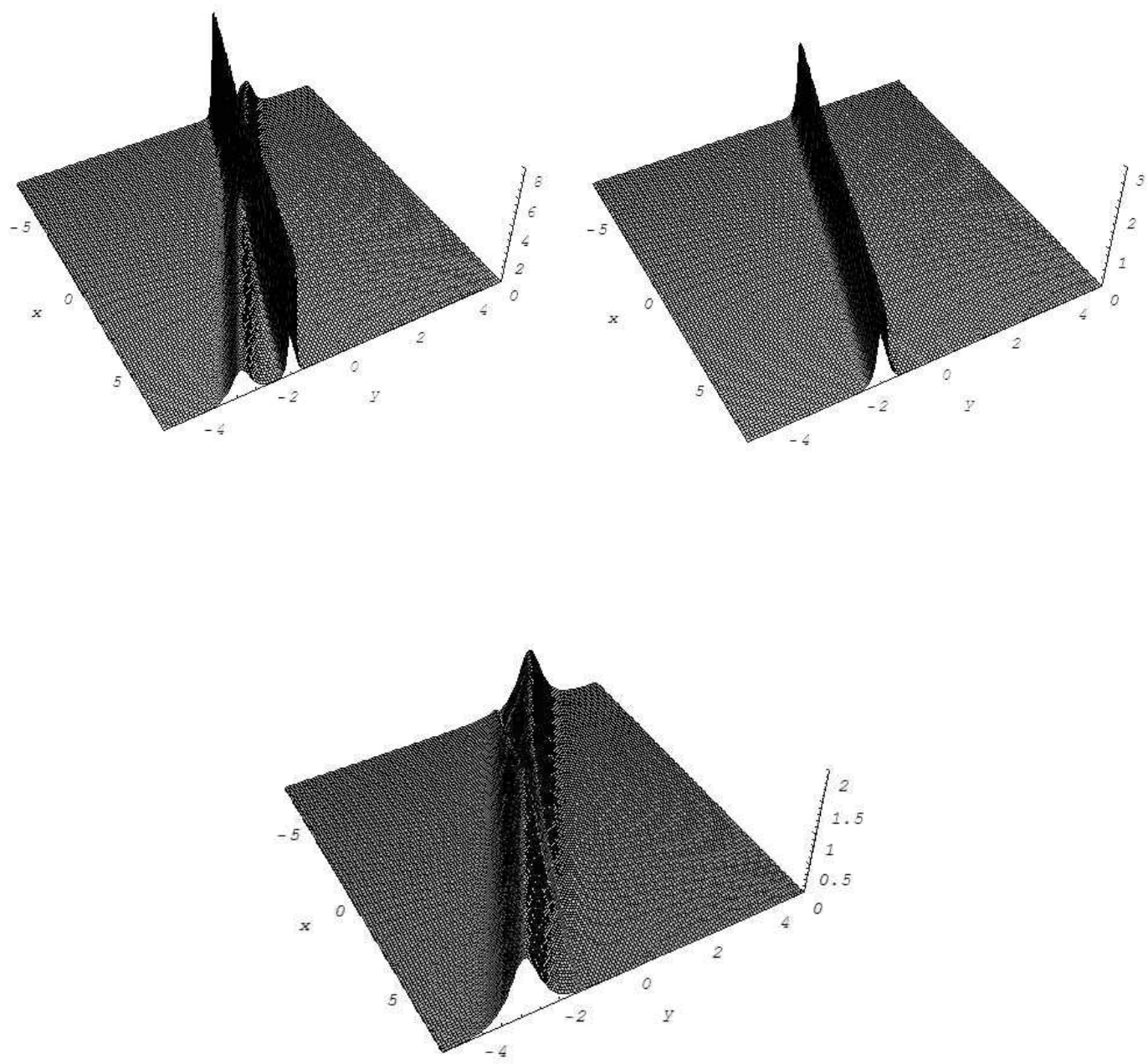

Figure 1. $(1,1,2)$-soliton solution. $p_{1}=2+2 i, s_{1}=-1+i, q_{1}=-2+i, r_{1}=1+i$, $\varepsilon=\delta=1$. The top left graph is $-L$, the top right graph is $S^{(1)}$ and the bottom graph is $S^{(2)}$ at $t=0$.

and $x_{1}=x, x_{2}=-i y, y_{1}=y-t, z_{1}=y-t$.

Figure 1 shows the $(1,1,2)$-soliton. In the field $S_{1}$ and $S_{2}$, there is the single soliton. However, each solitons in $S_{1}$ and $S_{2}$ interact through the field $L$. So the behavior of solitons looks like two-soliton solution, i.e., we see the phase shift in the region of the interaction (see the bottom graph in Fig.1). 


\section{$(2,2,4)$-soliton solution}

The $\tau$-functions of $(2,2,4)$-soliton solution are the following:

$$
f=\left|\begin{array}{cccccccc}
\varphi_{1} & \varphi_{1}^{(1)} & \varphi_{1}^{(2)} & \varphi_{1}^{(3)} & \psi_{1} & \psi_{1}^{(1)} & 0 & 0 \\
\varphi_{2} & \varphi_{2}^{(1)} & \varphi_{2}^{(2)} & \varphi_{2}^{(3)} & \psi_{2} & \psi_{2}^{(1)} & 0 & 0 \\
\varphi_{3} & \varphi_{3}^{(1)} & \varphi_{3}^{(2)} & \varphi_{3}^{(3)} & \psi_{3} & \psi_{3}^{(1)} & 0 & 0 \\
\varphi_{4} & \varphi_{4}^{(1)} & \varphi_{4}^{(2)} & \varphi_{4}^{(3)} & \psi_{4} & \psi_{4}^{(1)} & 0 & 0 \\
\phi_{1} & \phi_{1}^{(1)} & \phi_{1}^{(2)} & \phi_{1}^{(3)} & 0 & 0 & \chi_{1} & \chi_{1}^{(1)} \\
\phi_{2} & \phi_{2}^{(1)} & \phi_{2}^{(2)} & \phi_{2}^{(3)} & 0 & 0 & \chi_{2} & \chi_{2}^{(1)} \\
\phi_{3} & \phi_{3}^{(1)} & \phi_{3}^{(2)} & \phi_{3}^{(3)} & 0 & 0 & \chi_{3} & \chi_{3}^{(1)} \\
\phi_{4} & \phi_{4}^{(1)} & \phi_{4}^{(2)} & \phi_{4}^{(3)} & 0 & 0 & \chi_{4} & \chi_{4}^{(1)}
\end{array}\right|
$$




$$
\begin{aligned}
& \bar{h}=\left|\begin{array}{cccccccc}
\varphi_{1} & \varphi_{1}^{(1)} & \varphi_{1}^{(2)} & \psi_{1} & \psi_{1}^{(1)} & 0 & 0 & 0 \\
\varphi_{2} & \varphi_{2}^{(1)} & \varphi_{2}^{(2)} & \psi_{2} & \psi_{2}^{(1)} & 0 & 0 & 0 \\
\varphi_{3} & \varphi_{3}^{(1)} & \varphi_{3}^{(2)} & \psi_{3} & \psi_{3}^{(1)} & 0 & 0 & 0 \\
\varphi_{4} & \varphi_{4}^{(1)} & \varphi_{4}^{(2)} & \psi_{4} & \psi_{4}^{(1)} & 0 & 0 & 0 \\
\phi_{1} & \phi_{1}^{(1)} & \phi_{1}^{(2)} & 0 & 0 & \chi_{1} & \chi_{1}^{(1)} & \chi_{1}^{(2)} \\
\phi_{2} & \phi_{2}^{(1)} & \phi_{2}^{(2)} & 0 & 0 & \chi_{2} & \chi_{2}^{(1)} & \chi_{2}^{(2)} \\
\phi_{3} & \phi_{3}^{(1)} & \phi_{3}^{(2)} & 0 & 0 & \chi_{3} & \chi_{3}^{(1)} & \chi_{3}^{(2)} \\
\phi_{4} & \phi_{4}^{(1)} & \phi_{4}^{(2)} & 0 & 0 & \chi_{4} & \chi_{4}^{(1)} & \chi_{4}^{(2)}
\end{array}\right| \\
& \varphi_{1}=e^{\xi_{1}}, \quad \xi_{1}=p_{1} x_{1}+p_{1}^{2} x_{2}, \quad \varphi_{2}=e^{\xi_{2}}, \quad \xi_{2}=p_{2} x_{1}+p_{2}^{2} x_{2}, \\
& \varphi_{3}=e^{-\xi_{1}^{*}}, \quad-\xi_{1}^{*}=-p_{1}^{*} x_{1}+\left(-p_{1}^{*}\right)^{2} x_{2}, \quad \varphi_{4}=e^{-\xi_{2}^{*}}, \quad-\xi_{2}^{*}=-p_{2}^{*} x_{1}+\left(-p_{2}^{*}\right)^{2} x_{2}, \\
& \phi_{1}=e^{\theta_{1}}, \quad \theta_{1}=s_{1} x_{1}+s_{1}^{2} x_{2}, \quad \phi_{2}=e^{\theta_{2}}, \quad \theta_{2}=s_{2} x_{1}+s_{2}^{2} x_{2}, \\
& \phi_{3}=e^{-\theta_{1}^{*}}, \quad-\theta_{1}^{*}=-s_{1}^{*} x_{1}+\left(-s_{1}^{*}\right)^{2} x_{2}, \quad \phi_{4}=e^{-\theta_{2}^{*}}, \quad-\theta_{2}^{*}=-s_{2}^{*} x_{1}+\left(-s_{2}^{*}\right)^{2} x_{2}, \\
& \psi_{1}=a_{1} e^{\eta_{1}}, \quad \eta_{1}=q_{1} y_{1}+\eta_{10}, \quad \psi_{2}=a_{2} e^{\eta_{2}}, \quad \eta_{2}=q_{2} y_{1}+\eta_{20} \\
& \psi_{3}=a_{3} e^{-\eta_{1}^{*}}, \quad-\eta_{1}^{*}=-q_{1}^{*} y_{1}-\eta_{10}^{*}, \quad \psi_{4}=a_{4} e^{-\eta_{2}^{*}}, \quad-\eta_{2}^{*}=-q_{2}^{*} y_{1}-\eta_{20}^{*}, \\
& \chi_{1}=b_{1} e^{\zeta_{1}}, \quad \zeta_{1}=r_{1} z_{1}+\zeta_{10}, \quad \chi_{2}=b_{2} e^{\zeta_{2}}, \quad \zeta_{2}=r_{2} z_{1}+\zeta_{20}, \\
& \chi_{3}=b_{3} e^{-\zeta_{1}^{*}}, \quad-\zeta_{1}^{*}=-r_{1}^{*} z_{1}-\zeta_{10}^{*}, \quad \chi_{4}=b_{4} e^{-\zeta_{2}^{*}}, \quad-\zeta_{2}^{*}=-r_{2}^{*} z_{1}-\zeta_{20}^{*} \text {, } \\
& a_{1}=\frac{p_{2}-p_{1}}{q_{2}-q_{1}}\left(s_{1}-p_{1}\right)\left(s_{2}-p_{1}\right), \quad b_{1}=\frac{s_{2}-s_{1}}{r_{2}-r_{1}}\left(p_{1}-s_{1}\right)\left(p_{2}-s_{1}\right), \\
& a_{2}=\frac{p_{1}-p_{2}}{q_{1}-q_{2}}\left(s_{1}-p_{2}\right)\left(s_{2}-p_{2}\right), \quad b_{2}=\frac{s_{1}-s_{2}}{r_{1}-r_{2}}\left(p_{1}-s_{2}\right)\left(p_{2}-s_{2}\right), \\
& a_{3}=\varepsilon_{1} \frac{p_{1}+p_{1}^{*}}{q_{1}+q_{1}^{*}} \frac{p_{2}+p_{1}^{*}}{q_{2}+q_{1}^{*}}\left(s_{1}+p_{1}^{*}\right)\left(s_{2}+p_{1}^{*}\right), \quad b_{3}=\delta_{1} \frac{s_{1}+s_{1}^{*}}{r_{1}+r_{1}^{*}} \frac{s_{2}+s_{1}^{*}}{r_{2}+r_{1}^{*}}\left(s_{1}^{*}+p_{1}\right)\left(s_{1}^{*}+p_{2}\right), \\
& a_{4}=\varepsilon_{2} \frac{p_{1}+p_{2}^{*}}{q_{1}+q_{2}^{*}} \frac{p_{2}+p_{2}^{*}}{q_{2}+q_{2}^{*}}\left(s_{1}+p_{2}^{*}\right)\left(s_{2}+p_{2}^{*}\right), \quad b_{4}=\delta_{2} \frac{s_{1}+s_{2}^{*}}{r_{1}+r_{2}^{*}} \frac{s_{2}+s_{2}^{*}}{r_{2}+r_{2}^{*}}\left(s_{2}^{*}+p_{1}\right)\left(s_{2}^{*}+p_{2}\right), \\
& \varepsilon_{i}= \pm 1, \quad \delta_{i}= \pm 1(i=1,2) \text {, } \\
& \text { and } x_{1}=x, x_{2}=-i y, y_{1}=y-t, z_{1}=y-t \text {. }
\end{aligned}
$$

\section{Concluding Remarks}

We have derived two-component analogue of the two-dimensional long wave-short wave interaction equations. Then, we have presented Wronskian solutions to the system. Interestingly, the direction of a soliton on $S^{(1)}$ is different from one of a soliton on $S^{(2)}$. This is a special phenomenon in the case of two-dimensional vector soliton. We also found the interesting soliton interaction patterns including V-shape and breather-type soliton solutions.

Note that the generalization to $N$-component system is possible. In the $N$-component case, the solutions are constructed from $(N+1)$-component Wronskian of the $(N+1)$ component KP hierarchy.

Recently, another two-dimensional analogue of long wave-short wave interaction equations was derived asymptotically in [25]. The integrability and the existence of $N$-soliton 

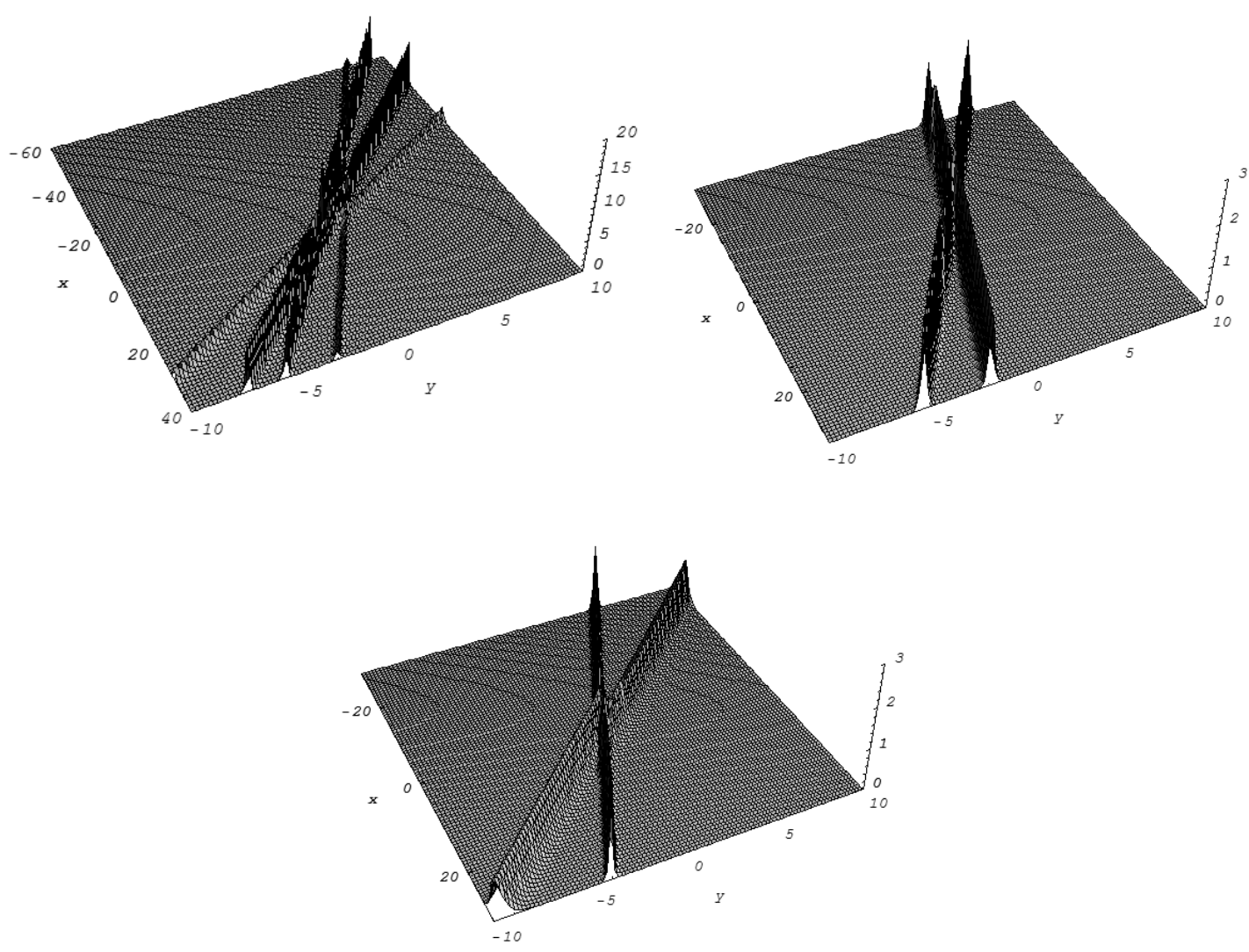

Figure 2. $(2,2,4)$-soliton solution. $p_{1}=2+2 i, p_{2}=1+3 i, p_{3}=2-2 i, p_{4}=1-3 i$, $s_{1}=-1+i, s_{2}=-2+3 i, s_{3}=-1-i, s_{4}=-2-3 i, q_{1}=-2+i, q_{2}=-3+2 i$, $q_{3}=-2-i, q_{4}=-3-2 i, r_{1}=1+i, r_{2}=1.5+i, r_{3}=1-i, r_{4}=1.5-i$, $\varepsilon_{1}=\varepsilon_{2}=\delta_{1}=\delta_{2}=1$. The top left graph is $-L$, the top right graph is $S^{(1)}$ and the bottom graph is $S^{(2)}$ at $t=3$.

solution of this system is unknown. The study of multi-component generalization of this system is also interesting.

The detail of analysis of soliton interaction will be presented in the forthcoming article.

\section{Acknowledgements}

We are grateful to Dr. Takayuki Tsuchida for many helpful discussions. K.M. acknowledges support from Faculty Research Council at the University of Texas-Pan American. 

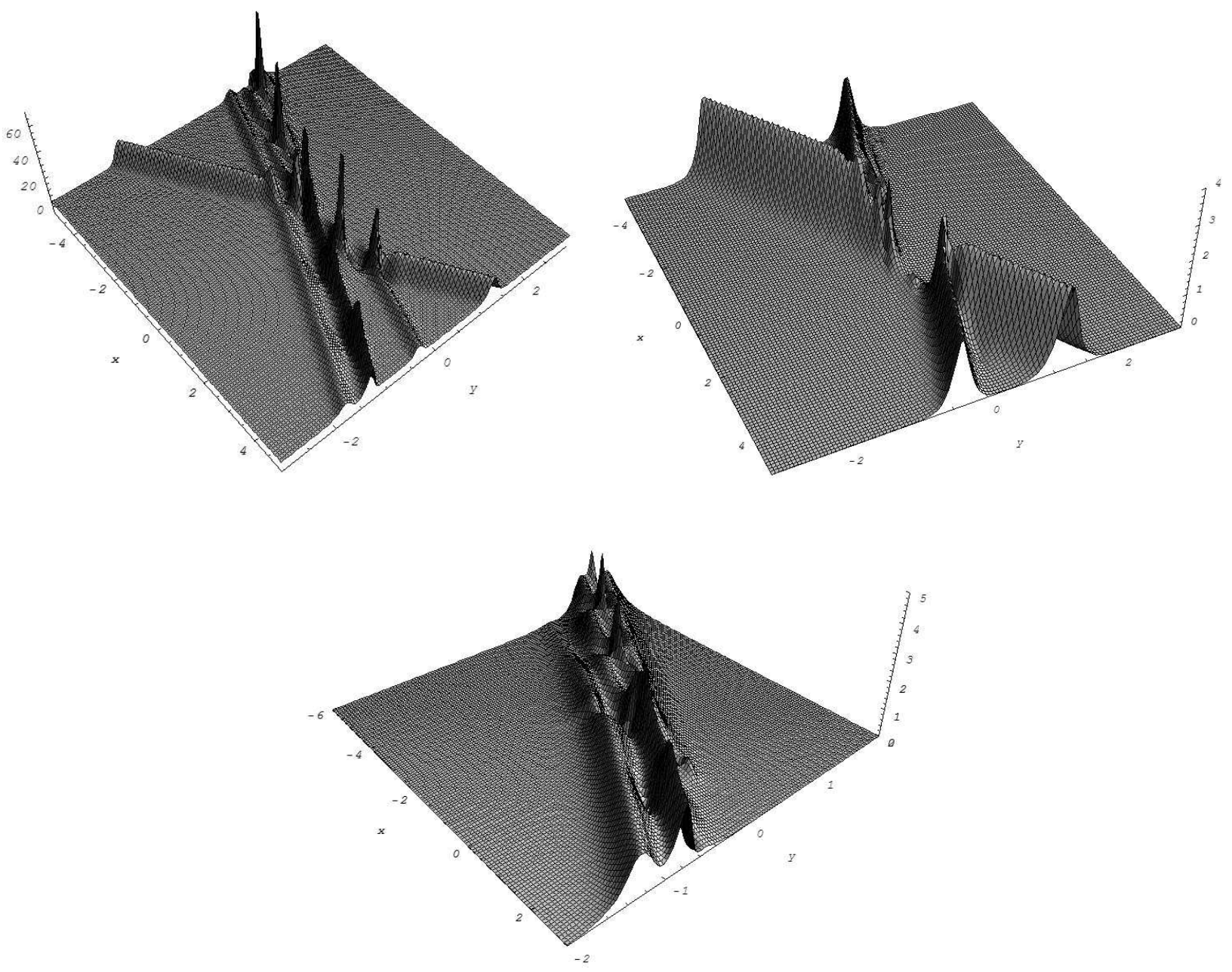

Figure 3. $(2,2,4)$-soliton solution. $p_{1}=2+3 i, p_{2}=3-i, p_{3}=2-3 i, p_{4}=3+i$, $s_{1}=2+2 i, s_{2}=4+2 i, s_{3}=2-2 i, s_{4}=-4-2 i, q_{1}=2+i, q_{2}=2.01+i$, $q_{3}=2-i, q_{4}=2.01-i, r_{1}=1+i, r_{2}=1.5+i, r_{3}=1-i, r_{4}=1.5-i$, $\varepsilon_{1}=\varepsilon_{2}=\delta_{1}=\delta_{2}=-1$. The top left graph is $-L$, the top right graph is $S^{(1)}$ and the bottom graph is $S^{(2)}$ at $t=0$.

\section{Appendix}

Consider the following three component Wronskian:

$\tau_{N M L}=$

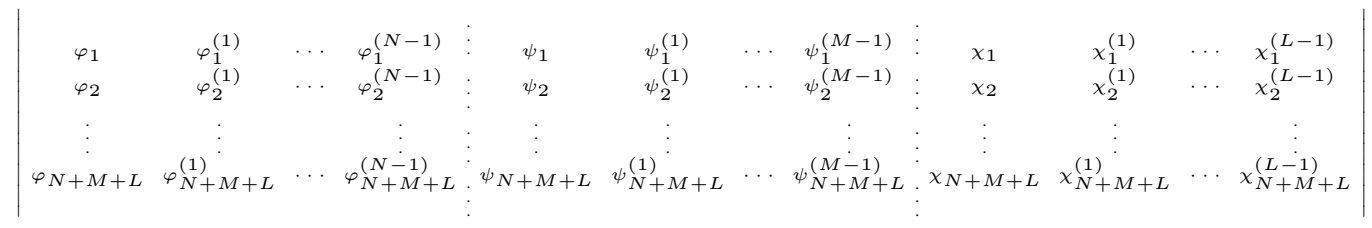


where $\varphi_{i}$ are functions of $x_{1}, x_{2}$ and satisfy

$$
\partial_{x_{2}} \varphi_{i}=\partial_{x_{1}}^{2} \varphi_{i}
$$

$\psi_{i}$ are arbitrary functions of $y_{1}, \chi_{i}$ are arbitrary functions of $z_{1}$, and

$$
\varphi_{i}^{(n)}=\partial_{x_{1}}^{n} \varphi_{i}, \quad \psi_{i}^{(n)}=\partial_{y_{1}}^{n} \psi_{i}, \quad \chi_{i}^{(n)}=\partial_{z_{1}}^{n} \chi_{i}
$$

This $\tau_{N M L}$ satisfies

$$
\begin{aligned}
& \left(D_{x_{1}}^{2}-D_{x_{2}}\right) \tau_{N+1, M-1, L} \cdot \tau_{N M L}=0 \\
& \left(D_{x_{1}}^{2}-D_{x_{2}}\right) \tau_{N+1, M, L-1} \cdot \tau_{N M L}=0 \\
& D_{x_{1}} D_{y_{1}} \tau_{N M L} \cdot \tau_{N M L}=2 \tau_{N+1, M-1, L} \tau_{N-1, M+1, L} \\
& D_{x_{1}} D_{z_{1}} \tau_{N M L} \cdot \tau_{N M L}=2 \tau_{N+1, M, L-1} \tau_{N-1, M, L+1}
\end{aligned}
$$

Setting

$f=\tau_{N M L}, \quad g=\tau_{N+1, M-1, L}, \quad \bar{g}=\tau_{N-1, M+1, L}, \quad h=\tau_{N+1, M, L-1}, \quad \bar{h}=\tau_{N-1, M, L+1}$,

these $\tau$-functions satisfy

$$
\begin{aligned}
& \left(D_{x_{1}}^{2}-D_{x_{2}}\right) g \cdot f=0 \\
& \left(D_{x_{1}}^{2}+D_{x_{2}}\right) \bar{g} \cdot f=0 \\
& \left(D_{x_{1}}^{2}-D_{x_{2}}\right) h \cdot f=0 \\
& \left(D_{x_{1}}^{2}+D_{x_{2}}\right) \bar{h} \cdot f=0 \\
& D_{x_{1}}\left(D_{y_{1}}+D_{z_{1}}\right) f \cdot f=2(g \bar{g}+h \bar{h}) .
\end{aligned}
$$

Applying the transformation of the dependent variables

$$
S_{1}=\frac{g}{f}, \quad \bar{S}_{1}=\frac{\bar{g}}{f}, \quad S_{2}=\frac{h}{f}, \quad \bar{S}_{2}=\frac{\bar{h}}{f}, \quad L=-(2 \log f)_{x_{1} x_{1}},
$$

we obtain

$$
\begin{aligned}
& \partial_{x_{1}}^{2} S_{1}-L S_{1}-\partial_{x_{2}} S_{1}=0 \\
& \partial_{x_{1}}^{2} \bar{S}_{1}-L \bar{S}_{1}+\partial_{x_{2}} \bar{S}_{1}=0 \\
& \partial_{x_{1}}^{2} S_{2}-L S_{2}-\partial_{x_{2}} S_{2}=0 \\
& \partial_{x_{1}}^{2} \bar{S}_{2}-L \bar{S}_{2}+\partial_{x_{2}} \bar{S}_{2}=0 \\
& -\left(\partial_{y_{1}}+\partial_{z_{1}}\right) L=2\left(S_{1} \bar{S}_{1}+S_{2} \bar{S}_{2}\right)_{x_{1}}
\end{aligned}
$$

Applying the change of independent variables

$$
x_{1}=x, \quad x_{2}=-i y, \quad y_{1}=y-t, \quad z_{1}=y-t,
$$

i.e.,

$$
\partial_{x}=\partial_{x_{1}}, \quad \partial_{y}=\partial_{y_{1}}+\partial_{z_{1}}-i \partial_{x_{2}}, \quad \partial_{t}=-\partial_{y_{1}}-\partial_{z_{1}},
$$

we obtain

$$
\begin{aligned}
& \partial_{x}^{2} S_{1}-L S_{1}-i\left(\partial_{t}+\partial_{y}\right) S_{1}=0, \\
& \partial_{x}^{2} \bar{S}_{1}-L \bar{S}_{1}+i\left(\partial_{t}+\partial_{y}\right) \bar{S}_{1}=0, \\
& \partial_{x}^{2} S_{2}-L S_{2}-i\left(\partial_{t}+\partial_{y}\right) S_{2}=0, \\
& \partial_{x}^{2} \bar{S}_{2}-L \bar{S}_{2}+i\left(\partial_{t}+\partial_{y}\right) \bar{S}_{2}=0, \\
& L_{t}=2\left(S_{1} \bar{S}_{1}+S_{2} \bar{S}_{2}\right)_{x} .
\end{aligned}
$$


In the above solution, we consider the replacements of $N, M$ and $L$ by

$$
N \rightarrow 2 N, \quad M \rightarrow N, \quad L \rightarrow N
$$

i.e., consider

$f=\tau_{2 N, N, N} \quad g=\tau_{2 N+1, N-1, N} \quad \bar{g}=\tau_{2 N-1, N+1, N} \quad h=\tau_{2 N+1, N, N-1} \quad \bar{h}=\tau_{2 N-1, N, N+1}$.

Setting

$$
\psi_{2 N+1}=\psi_{2 N+2}=\cdots=\psi_{4 N}=0, \quad \chi_{1}=\chi_{2}=\cdots=\chi_{2 N}=0,
$$

we have

$\tau_{2 N+n+m, N-n, N-m}=$

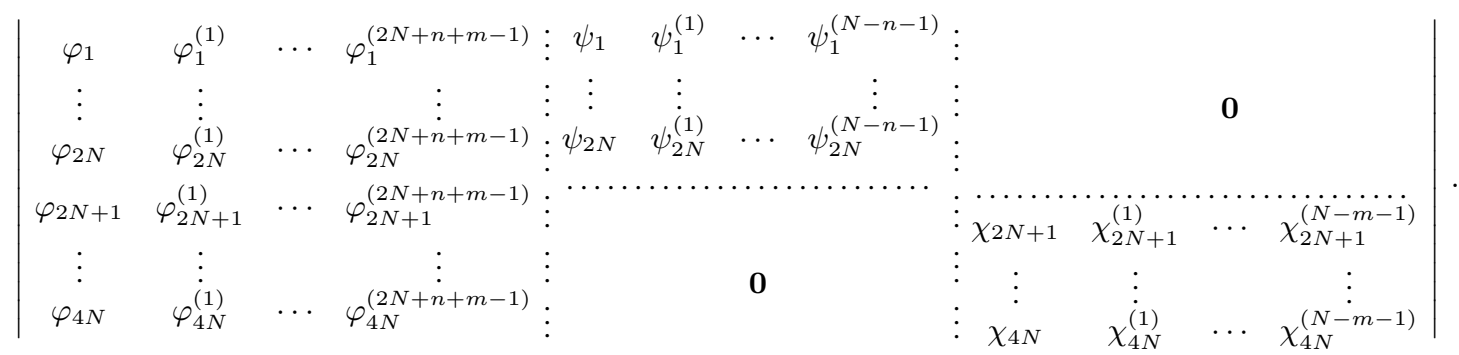

Note that this determinant is 0 if $N-n>2 N$ or $N-m>2 N$. Consider the following table of non-zero $\tau$-functions:

$$
\begin{array}{cccccc}
m=N & \tau_{2 N, 2 N, 0} & \tau_{2 N+1,2 N-1,0} & \cdots & \tau_{4 N-1,1,0} & \tau_{4 N, 0,0} \\
m=N-1 & \tau_{2 N-1,2 N, 1} & \tau_{2 N, 2 N-1,1} & \cdots & \tau_{4 N-2,1,1} & \tau_{4 N-1,0,1} \\
& \vdots & \vdots & & \vdots & \vdots \\
m=-N+1 & \tau_{1,2 N, 2 N-1} & \tau_{2,2 N-1,2 N-1} & \cdots & \tau_{2 N, 1,2 N-1} & \tau_{2 N+1,0,2 N-1} \\
m=-N & \tau_{0,2 N, 2 N} & \tau_{1,2 N-1,2 N} & \cdots & \tau_{2 N-1,1,2 N} & \tau_{2 N, 0,2 N} \\
& n=-N & n=-N+1 & & n=N-1 & n=N
\end{array}
$$

The $\tau$-function in the center on the table is corresponding to $f$ :

$\begin{array}{ccccccc}\tau_{2 N, 2 N, 0} & \tau_{2 N+1,2 N-1,0} & \cdots & \cdots & \cdots & \tau_{4 N-1,1,0} & \tau_{4 N, 0,0} \\ \tau_{2 N-1,2 N, 1} & \tau_{2 N, 2 N-1,1} & \cdots & \cdots & \cdots & \tau_{4 N-2,1,1} & \tau_{4 N-1,0,1} \\ \vdots & \vdots & & h & & \vdots & \vdots \\ \vdots & \vdots & \bar{g} & f & g & \vdots & \vdots \\ \vdots & \vdots & & \bar{h} & & \vdots & \vdots \\ \tau_{1,2 N, 2 N-1} & \tau_{2,2 N-1,2 N-1} & \cdots & \cdots & \cdots & \tau_{2 N, 1,2 N-1} & \tau_{2 N+1,0,2 N-1} \\ \tau_{0,2 N, 2 N} & \tau_{1,2 N-1,2 N} & \cdots & \cdots & \cdots & \tau_{2 N-1,1,2 N} & \tau_{2 N, 0,2 N}\end{array}$

Now we want to find the condition of complex conjugacy $\left(f:\right.$ real, $\bar{g}=g^{*}, \bar{h}=h^{*}$ where $*$ means complex conjugate). For the function $\tau_{2 N+n+m, N-n, N-m}$, the bilinear equations of two-dimensional Toda lattice

$$
\begin{aligned}
& D_{x_{1}} D_{y_{1}} \tau_{2 N+n+m, N-n, N-m} \cdot \tau_{2 N+n+m, N-n, N-m} \\
& \quad=2 \tau_{2 N+n+m+1, N-n-1, N-m} \tau_{2 N+n+m-1, N-n+1, N-m} \\
& D_{x_{1}} D_{z_{1}} \tau_{2 N+n+m, N-n, N-m} \cdot \tau_{2 N+n+m, N-n, N-m} \\
& \quad=2 \tau_{2 N+n+m+1, N-n, N-m-1} \tau_{2 N+n+m-1, N-n, N-m+1}
\end{aligned}
$$


are satisfied. Since the function $\tau_{4 N, 0,0}$ depends on only $x_{1}, x_{2}$, the following bilinear equations are satisfied:

$$
\begin{array}{r}
D_{x_{1}} D_{y_{1}} \frac{\tau_{2 N+n+m, N-n, N-m}}{\tau_{4 N, 0,0}} \cdot \frac{\tau_{2 N+n+m, N-n, N-m}}{\tau_{4 N, 0,0}} \\
=2 \frac{\tau_{2 N+n+m+1, N-n-1, N-m}}{\tau_{4 N, 0,0}} \frac{\tau_{2 N+n+m-1, N-n+1, N-m}}{\tau_{4 N, 0,0}}, \\
D_{x_{1}} D_{z_{1}} \frac{\tau_{2 N+n+m, N-n, N-m}}{\tau_{4 N, 0,0}} \cdot \frac{\tau_{2 N+n+m, N-n, N-m}}{\tau_{4 N, 0,0}} \\
=2 \frac{\tau_{2 N+n+m+1, N-n, N-m-1}}{\tau_{4 N, 0,0}} \frac{\tau_{2 N+n+m-1, N-n, N-m+1}}{\tau_{4 N, 0,0}} .
\end{array}
$$

\begin{tabular}{|c|c|c|c|c|c|c|}
\hline$\tau_{2 N, 2 N, 0}$ & $\underline{\tau_{2 N+1,2 N-1,0}}$ & & $\cdots$ & $\cdots$ & $\underline{\tau_{4 N-1,1,0}}$ & 1 \\
\hline $\begin{array}{c}\tau_{4 N, 0,0} \\
\tau_{2 N-1,2 N, 1} \\
\end{array}$ & $\begin{array}{c}\tau_{4 N, 0,0} \\
\tau_{2 N, 2 N-1,1} \\
\end{array}$ & & & & $\begin{array}{c}\tau_{4 N, 0,0} \\
\tau_{4 N-2,1,1} \\
\end{array}$ & $\tau_{4 N-1,0,1}$ \\
\hline$\tau_{4 N, 0,0}$ & $\tau_{4 N, 0,0}$ & & & & $\tau_{4 N, 0,0}$ & $\tau_{4 N, 0,0}$ \\
\hline : & : & & $h$ & & : & : \\
\hline & & & $\tau_{4 N, 0,0}$ & & & \\
\hline$\vdots$ & $\vdots$ & $\bar{g}$ & $f$ & $g$ & $\vdots$ & $\vdots$ \\
\hline$:$ & $:$ & $\tau_{4 N, 0,0}$ & $\tau_{4 N, 0,0}$ & $\tau_{4 N, 0,0}$ & $:$ & $:$ \\
\hline 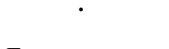 & 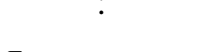 & & $\overline{\tau_{4 N, 0,0}}$ & & & \\
\hline$\tau_{1,2 N, 2 N-1}$ & $\underline{\tau_{2,2 N}-1,2 N-1}$ & & $\ldots$ & & $\underline{\tau_{2 N, 1,2 N-1}}$ & $\tau_{2 N+1,0,2 N-1}$ \\
\hline $\begin{array}{c}\tau_{4 N, 0,0} \\
\tau_{0,2 N, 2 N}\end{array}$ & $\begin{array}{c}\tau_{4 N, 0,0} \\
\tau_{1,2 N-1,2 N}\end{array}$ & & & & $\begin{array}{c}\tau_{4 N, 0,0} \\
\tau_{2 N-1,1,2 N}\end{array}$ & $\begin{array}{c}\tau_{4 N, 0,0} \\
\tau_{2 N}, 2 N\end{array}$ \\
\hline$\tau_{4 N, 0,0}$ & $\tau_{4 N, 0,0}$ & & & & $\tau_{4 N, 0,0}$ & $\tau_{4 N, 0,0}$ \\
\hline
\end{tabular}

The table of solutions of these bilinear equations are as follows:

Since the function $\tau_{0,2 N, 2 N}$ does not depend on $x_{1}$, the following bilinear equations are also satisfied:

$$
\begin{array}{r}
D_{x_{1}} D_{y_{1}} \frac{\tau_{2 N+n+m, N-n, N-m}}{\tau_{0,2 N, 2 N}} \cdot \frac{\tau_{2 N+n+m, N-n, N-m}}{\tau_{0,2 N, 2 N}} \\
=2 \frac{\tau_{2 N+n+m+1, N-n-1, N-m}}{\tau_{0,2 N, 2 N}} \frac{\tau_{2 N+n+m-1, N-n+1, N-m}}{\tau_{0,2 N, 2 N}}, \\
D_{x_{1}} D_{z_{1}} \frac{\tau_{2 N+n+m, N-n, N-m}}{\tau_{0,2 N, 2 N}} \cdot \frac{\tau_{2 N+n+m, N-n, N-m}}{\tau_{0,2 N, 2 N}} \\
=2 \frac{\tau_{2 N+n+m+1, N-n, N-m-1}}{\tau_{0,2 N, 2 N}} \frac{\tau_{2 N+n+m-1, N-n, N-m+1}}{\tau_{0,2 N, 2 N}} .
\end{array}
$$


The table of solutions of these bilinear equations are as follows:

\begin{tabular}{|c|c|c|c|c|c|c|}
\hline$\tau_{2 N, 2 N, 0}$ & $\tau_{2 N+1,2 N-1,0}$ & $x_{0}>0$ & & $\cdots$ & $\tau_{4 N-1,1,0}$ & $\tau_{4 N, 0,0}$ \\
\hline $\begin{array}{c}\tau_{0,2 N, 2 N} \\
\tau_{2 N-1,2 N, 1}\end{array}$ & $\begin{array}{c}\tau_{0,2 N, 2 N} \\
\tau_{2 N, 2 N-1,1}\end{array}$ & $\ldots$ & & & $\begin{array}{c}\tau_{0,2 N, 2 N} \\
\tau_{4 N-2,1,1}\end{array}$ & $\begin{array}{c}\tau_{0,2 N, 2 N} \\
\tau_{4 N-1,0,1}\end{array}$ \\
\hline$\tau_{0,2 N, 2 N}$ & $\tau_{0,2 N, 2 N}$ & & & & $\tau_{0,2 N, 2 N}$ & $\tau_{0,2 N, 2 N}$ \\
\hline 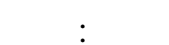 & : & & $h$ & & : & : \\
\hline & & & $\tau_{0,2 N, 2 N}$ & & & \\
\hline : & : & $\bar{g}$ & $f$ & $g$ & : & : \\
\hline & & $\tau_{0,2 N, 2 N}$ & $\overline{\tau_{0,2 N} \underset{-}{2}, 2 N}$ & $\tau_{0,2 N, 2 N}$ & & \\
\hline : & : & & $\bar{h}$ & & : & : \\
\hline & & & $\tau_{0,2 N, 2 N}$ & & & \\
\hline$\tau_{1,2 N, 2 N-1}$ & $\tau_{2,2 N-1,2 N-1}$ & & , & $\ldots$ & $\tau_{2 N, 1,2 N-1}$ & $\tau_{2 N+1,0,2 N-}$ \\
\hline$\tau_{0,2 N, 2 N}$ & $\tau_{0,2 N, 2 N}$ & & & & $\tau_{0,2 N, 2 N}$ & $\tau_{0,2 N, 2 N}$ \\
\hline $0,218,21$ & $\tau_{1,2 N-1,2 N}$ & $\ldots$ & $\ldots$ & & $\tau_{2 N-1,1,2 N}$ & $\tau_{2 N, 0,2 N}$ \\
\hline & $\tau_{0,2 N, 2 N}$ & & & & $\tau_{0,2 N, 2 N}$ & $\tau_{0,2 N, 2 N}$ \\
\hline
\end{tabular}

Consider the case in which the relations

$$
\frac{\tau_{4 N-1,1,0}}{\tau_{4 N, 0,0}}=\left(\frac{\tau_{1,2 N-1,2 N}}{\tau_{0,2 N, 2 N}}\right)^{*},
$$

and

$$
\frac{\tau_{4 N-1,0,1}}{\tau_{4 N, 0,0}}=\left(\frac{\tau_{1,2 N, 2 N-1}}{\tau_{0,2 N, 2 N}}\right)^{*},
$$

are satisfied. If we choose elements in the solution in the section 3 as $\varphi_{i}, \psi_{i}, \chi_{i}$, these relations are satisfied, and we also notice that the relations

$$
\begin{aligned}
& \frac{f}{\tau_{4 N, 0,0}}=\left(\frac{f}{\tau_{0,2 N, 2 N}}\right)^{*}, \\
& \frac{g}{\tau_{4 N, 0,0}}=\left(\frac{\bar{g}}{\tau_{0,2 N, 2 N}}\right)^{*}, \\
& \frac{h}{\tau_{4 N, 0,0}}=\left(\frac{\bar{h}}{\tau_{0,2 N, 2 N}}\right)^{*},
\end{aligned}
$$

are satisfied. From these relations, we conclude that the complex conjugacy condition

$$
\begin{gathered}
\left(\frac{g}{f}\right)^{*}=\frac{\bar{g}}{f}, \quad\left(\frac{h}{f}\right)^{*}=\frac{\bar{h}}{f}, \\
f \mathcal{G}: \text { real }
\end{gathered}
$$

where $\mathcal{G}$ is an exponential factor which is a gauge function, is satisfied.

\section{References}

[1] M. J. Ablowitz, B. Prinari and A. D. Trubatch: Discrete and Continuous Nonlinear Schrödinger Systems (Cambridge University Press) 2004.

[2] Manakov S V 1974 Sov. Phys. JETP 38, 248

[3] Radhakrishnan R, Lakshmanan M, and Hietarinta J 1997 Phys. Rev. E 56, 2213

[4] Tsuchida T 2004 Prog. Theor. Phys. 111, 151 
[5] Kanna T and Lakshmanan M 2001 Phys. Rev. Lett. 86, 5043

[6] Kanna T and Lakshmanan M 2003 Phys. Rev. E 67, 046617

[7] Jakubowski M H, Steiglitz K and Squier R 1998 Phys. Rev. E 58, 6752

[8] Steiglitz K 2000 Phys. Rev. E. 63, 016608

[9] Veselov A P 2003 Phys. Lett. A 314, 214

[10] Ablowitz M J, Prinari B and Trubatch A D 2004 Inv. Probl. 20, 1217

[11] Asano N 1974 J. Phys. Soc. Jpn. 36, 861

[12] Kivshar Y 1992 Opt. Lett. 17, 1322.

[13] Benney D J 1976 Studies Appl. Math. 55, 93

[14] Yajima N and Oikawa M 1976 Prog. Theor. Phys. 56, 1719

[15] Oikawa M, Okamura M and Funakoshi M 1989 J. Phys. Soc. Jpn. 58, 4416

[16] Melnikov V K 1983 Lett. Math. Phys. 7, 129

[17] Lai D W C and Chow K W 1999 J. Phys. Soc. Jpn. 68, 1847

[18] Radha R, Kumar C S, Lakshmanan M, Tang X Y and Lou S Y 2005 J. Phys. A:Math. Gen. 38, 9649

[19] Yurova M 2006 arXiv:nlin.SI/0612041

[20] Berkela Y 2002 Proceedings of Inst. Math. NAS of Ukraine 43, 296

[21] Date E, Jimbo M, Kashiwara M and Miwa T 1981 J. Phys. Soc. Jpn. 50, 3806

[22] Date E, Jimbo M, Kashiwara M and Miwa T 1982 Publ. Res. Inst. Math. Sci. 18, 1077

[23] Kac V G and van de Leur J W 2003 J. Math. Phys. 44, 3245

[24] Hirota R 2004 The Direct Method in Soliton Theory (Cambridge University Press)

[25] Aguero M, Frantzeskakis D J and Kevrekidis P G 2006 J. Phys. A:Math. Gen. 39, 7705 